\title{
Risk for Severe COVID-19 Illness Among Health Care Workers Who Work Directly with Patients
}

J Gen Intern Med 35(9):2804-6

DOI: $10.1007 /$ s11606-020-05992-y

(C) Society of General Internal Medicine 2020

\section{INTRODUCTION}

A recent study from the Centers for Disease Control found that health care workers in the USA accounted for $19 \%$ of confirmed COVID-19 cases with information on occupation. ${ }^{1}$ At the same time, evidence is mounting that pre-existing conditions such as cardiovascular disease, diabetes, hypertension, and obesity, as well as older age, are associated with a higher risk of hospitalization, admission to an intensive care unit, and death among those with COVID-19. ${ }^{2}$ Health care workers who have direct contact with patients not only have an increased likelihood of being exposed to COVID-19, but those who are older or who have high-risk comorbidities are at particular risk of adverse COVID-19 outcomes. In the USA, $8.8 \%$ of physicians and $3.8 \%$ of registered nurses are aged $\geq$ 65 years. $^{3}$ The objective of this study is to estimate the prevalence of high-risk comorbidities and older age for five types of health care workers, focusing on those who have direct contact with patients. The study also examines the sociodemographic characteristics of health care workers who are at higher risk for adverse COVID-19 outcomes.

\section{METHODS}

This study used pooled data on adults aged $\geq 18$ years from the 2017 and 2018 National Health Interview Surveys (NHIS) ( $n$ $=52,159$ ). The NHIS are nationally representative of the noninstitutionalized civilian population. A subsample was created of individuals who reported physical or hands-on contact with patients as part of their routine work and whose occupation was health care practitioner or technician (Standard Occupational Classification (SOC) code 29) or health care support (SOC code 31) $(n=1814) .{ }^{4}$ Practitioner and technician occupation categories were further defined by the entry level education required for the occupation (see Tables 1 and 2). ${ }^{5}$

COVID-19 high-risk comorbidity variables available from the NHIS were BMI $\geq 40$, cancer in the past 3 years, cardiovascular disease, chronic lung disease or an asthma attack in

Received May 22, 2020

Accepted June 12, 2020

Published online June 24, 2020 the past year, current smoker, diabetes, kidney disease, and liver disease. ${ }^{2}$ Direct patient care health care workers were categorized as being at higher risk of adverse COVID-19 outcomes ("higher-risk health care workers") if they had $\geq 1$ high-risk comorbidity or if they were aged $\geq 65$ years $(n=$ $745)$. Descriptive statistics were calculated and Pearson design-based $F$-tests were used to determine whether percentages differed significantly across occupation categories. All estimates were weighted and accounted for the NHIS complex sampling design.

\section{RESULTS}

Of the $3.8 \%$ of US adults who worked directly with patients as health care workers, $38.6 \%$ were higher risk. Among direct patient care health care workers, the prevalence of high-risk comorbidities, older age, and higher-risk status differed significantly across occupation categories (Table 1). Among the subsample of higher-risk health care workers, $67.5 \%$ had 1 high-risk comorbidity, $28.3 \%$ had $\geq 2$ high-risk comorbidities, and $10.8 \%$ were aged $\geq 65$ years. Of this subsample, $52.5 \%$ worked in support roles, $54.0 \%$ worked in hospitals or nursing homes, $74.9 \%$ were women, $64.4 \%$ were white, $17.8 \%$ were African American, and 9.9\% were Latino. Among higher-risk health care workers, there were significant differences across occupation categories in work setting, gender, race/ethnicity, health insurance status, and financial resources (Table 2).

\section{DISCUSSION}

The findings of this study suggest that more than a third of individuals in health care occupations who work directly with patients are at an elevated risk of adverse COVID-19 outcomes if they become ill. Additionally, a quarter of higherrisk health care workers have multiple high-risk comorbidities, further increasing the likelihood of adverse COVID-19 outcomes. More than half of higher-risk health care workers work in hospitals or nursing homes, settings with high levels of exposure to COVID-19. These findings highlight the crucial importance of personal protective equipment for those who work directly with patients across health care occupations and locations of care. Furthermore, health care practitioners and technicians with lower education and those in health care support occupations are more likely to have high-risk comorbidities as well as strained financial resources. Assuming that 
Table 1 Prevalence of High-Risk Characteristics for Adverse COVID-19 Outcomes Among Health Care Workers Who Work Directly with Patients, by Occupation Category $(n=1814)$

\begin{tabular}{|c|c|c|c|c|c|c|}
\hline & $\begin{array}{l}\text { Doctors and } \\
\text { other } \\
\text { clinicians } \\
(n=252)\end{array}$ & $\begin{array}{l}\text { PA/NP, } \\
\text { therapists, } \\
\text { and others with } \\
\text { Master's degree } \\
(n=151)\end{array}$ & $\begin{array}{l}\text { Nurses, } \\
\text { nutritionists, } \\
\text { and others with } \\
\text { Bachelor's degree } \\
(n=433)\end{array}$ & $\begin{array}{l}\text { LPNs, EMTs, } \\
\text { and others } \\
\text { with less than } \\
\text { a Bachelor's } \\
\text { degree } \\
(n=462)\end{array}$ & $\begin{array}{l}\text { Home health } \\
\text { aides, } \\
\text { medical } \\
\text { assistants, } \\
\text { and other } \\
\text { support } \\
\text { roles } \\
(n=516)\end{array}$ & \\
\hline & $\begin{array}{l}\text { Weighted \% } \\
\text { (95\% CI) }\end{array}$ & $\begin{array}{l}\text { Weighted \% } \\
\text { (95\% CI) }\end{array}$ & $\begin{array}{l}\text { Weighted \% } \\
\text { (95\% CI) }\end{array}$ & $\begin{array}{l}\text { Weighted \% } \\
(95 \% \text { CI) }\end{array}$ & $\begin{array}{l}\text { Weighted \% } \\
\text { (95\% CI) }\end{array}$ & $\begin{array}{l}p \\
\text { value }\end{array}$ \\
\hline 1 or more high-risk comorbidity & $\begin{array}{l}29.6(23.5- \\
36.4)\end{array}$ & $25.4(18.5-33.8)$ & $32.1(26.9-37.7)$ & $\begin{array}{l}45.5(39.7- \\
51.5)\end{array}$ & $41.2(36.2-46.4)$ & $<.000$ \\
\hline Age $\geq 65$ years old & $9.2(6.3-13.3)$ & $1.5(0.7-3.5)$ & $4.3(2.7-6.8)$ & $3.0(1.8-4.9)$ & $3.2(2.0-5.1)$ & $<.000$ \\
\hline $\begin{array}{l}1 \text { or more high-risk comorbidity or } \\
\text { age } \geq 65\end{array}$ & $\begin{array}{l}32.4(26.1- \\
39.4)\end{array}$ & $26.6(19.6-35.0)$ & $33.8(28.7-39.4)$ & $\begin{array}{l}46.8(40.9- \\
52.7)\end{array}$ & $42.6(37.5-47.8)$ & $<.000$ \\
\hline
\end{tabular}

PA physician assistant, NP nurse practitioner, LPN licensed practical nurse, EMT emergency medical technician. Of individuals in the health care sector who worked directly with patients, $14.8 \%$ were doctors or other clinicians; $8.4 \%$ were physician assistants, nurse practitioners, therapists, or others with Master's degrees; $24.4 \%$ were nurses, nutritionists, or others with Bachelor's degrees; $24.6 \%$ were licensed practical nurses, EMTs, or others with less than a Bachelor's degree; and 27.9\% were home health aides or medical assistants. Analyses were weighted and accounted for the NHIS complex sampling design

Table 2 Work Setting, Demographic, and Socioeconomic Characteristics of Health Care Workers Who Work Directly with Patients and Are at Higher Risk of Adverse COVID-19 Outcomes, by Occupation Category $(n=745)$

\begin{tabular}{|c|c|c|c|c|c|c|}
\hline & $\begin{array}{l}\text { Doctors and } \\
\text { other } \\
\text { clinicians }(n= \\
\text { 86) }\end{array}$ & $\begin{array}{l}\text { PA/NP, therapists, } \\
\text { and others with } \\
\text { Master's degree ( } n \\
=50 \text { ) }\end{array}$ & $\begin{array}{l}\text { Nurses, nutritionists, } \\
\text { and others with } \\
\text { Bachelor's degree ( } n \\
=149 \text { ) }\end{array}$ & $\begin{array}{l}\text { LPNs, EMTs, and } \\
\text { others with less than } \\
\text { a Bachelor's degree } \\
(n=227)\end{array}$ & $\begin{array}{l}\text { Home health aides, } \\
\text { medical assistants, } \\
\text { and other support } \\
\text { roles }(n=233)\end{array}$ & \\
\hline & $\begin{array}{l}\text { Weighted \% } \\
\text { (95\% CI) }\end{array}$ & $\begin{array}{l}\text { Weighted \% (95\% } \\
\text { CI) }\end{array}$ & $\begin{array}{l}\text { Weighted \% (95\% } \\
\text { CI) }\end{array}$ & $\begin{array}{l}\text { Weighted \% (95\% } \\
\text { CI) }\end{array}$ & $\begin{array}{l}\text { Weighted \% (95\% } \\
\text { CI) }\end{array}$ & $\begin{array}{l}p \\
\text { value }\end{array}$ \\
\hline \multicolumn{7}{|l|}{ Work setting } \\
\hline Hospital & $\begin{array}{l}24.7(15.1- \\
37.8)\end{array}$ & $64.1(46.9-78.4)$ & $65.3(55.4-74.0)$ & $44.9(36.8-53.3)$ & $13.4(9.2-19.2)$ & $\begin{array}{l}< \\
.000\end{array}$ \\
\hline Nursing home & $1.9(0.5-7.4)$ & $1.4(0.2-9.5)$ & $2.6(1.0-6.8)$ & $14.5(9.7-21.1)$ & $34.5(27.7-42.1)$ & $\begin{array}{l}<. \\
.000\end{array}$ \\
\hline Female & $\begin{array}{l}28.1(17.8- \\
41.4)\end{array}$ & $79.0(63.5-89.0)$ & $83.7(74.5-90.2)$ & $77.8(69.6-84.4)$ & $87.6(81.2-92.0)$ & $<.000$ \\
\hline \multicolumn{7}{|l|}{ Race/ethnicity } \\
\hline White (non-Latino) & $\begin{array}{l}73.3(59.6- \\
83.5)\end{array}$ & $73.5(54.6-86.5)$ & $56.2(45.1-66.7)$ & $73.7(65.7-80.4)$ & $55.8(47.6-63.7)$ & $\begin{array}{l}< \\
.000\end{array}$ \\
\hline $\begin{array}{l}\text { African American } \\
\text { (non-Latino) }\end{array}$ & $7.2(2.5-19.2)$ & $2.7(0.4-16.8)$ & $20.0(12.7-32.7)$ & $14.5(9.7-21.3)$ & $25.8(19.4-33.5)$ & \\
\hline Latino & $1.7(0.4-7.0)$ & $6.3(1.6-21.9)$ & $6.8(2.4-17.9)$ & $10.5(6.2-17.0)$ & $15.6(10.2-23.1)$ & \\
\hline Other & $17.8(9.4-31.4)$ & $17.5(7.0-37.5)$ & $16.0(9.3-26.2)$ & $1.3(0.3-4.9)$ & $2.8(1.1-7.3)$ & \\
\hline $\begin{array}{l}\text { Has health } \\
\text { insurance }\end{array}$ & $\begin{array}{l}98.4(92.9- \\
99.7)\end{array}$ & 100 & 98.7 (95.4-99.6) & $93.5(87.7-96.6)$ & $85.8(79.8-90.2)$ & $\begin{array}{l}< \\
.000\end{array}$ \\
\hline \multicolumn{7}{|c|}{ Family financial resources } \\
\hline $\begin{array}{l}\text { Income }<200 \% \text { of } \\
\text { federal poverty line }\end{array}$ & $0.4(0.1-2.9)$ & $1.0(0.1-6.8)$ & $4.5(2.0-9.7)$ & $12.2(8.2-18.0)$ & $36.9(29.4-45.1)$ & $\begin{array}{l}< \\
.000\end{array}$ \\
\hline $\begin{array}{l}\text { Could not afford } \\
\text { prescription } \\
\text { medication, past } 12 \\
\text { months }\end{array}$ & $0.7(0.1-4.6)$ & $1.0(0.1-6.8)$ & $5.6(2.9-10.6)$ & $7.3(4.3-12.0)$ & $17.5(12.0-24.7)$ & $\begin{array}{l}< \\
.000\end{array}$ \\
\hline $\begin{array}{l}\text { Worried food } \\
\text { would run out, past } \\
30 \text { days }\end{array}$ & 0 & 0 & $2.0(0.1-6.9)$ & $12.2(8.1-18.0)$ & $26.6(20.3-34.1)$ & $<.000$ \\
\hline
\end{tabular}


the estimates from the pooled 2017 and 2018 NHIS data offer reasonable estimates for 2020 , the findings highlight the precarious position of health care workers who hold these jobs and the difficult trade-off they may face in deciding whether to risk their health or to risk losing their livelihood.

Diane M. Gibson, Ph.D. ${ }^{1}$

Jessica Greene, Ph.D. ${ }^{1}$

${ }^{1}$ Marxe School of Public and International Affairs, Baruch College - City University of New York, New York, NY, USA

Corresponding Author: Diane M. Gibson, Ph.D.; Marxe School of Public and International Affairs, Baruch College-City University of New York, New York, NY, USA (e-mail: diane.gibson@baruch.cuny.edu).

\section{Compliance with Ethical Standards:}

Conflict of Interest: The authors declare that they do not have a conflict of interest.

\section{REFERENCES}

1. CDC COVID-19 Response Team. Characteristics of Health Care Personnel with COVID-19 - United States, February 12-April 9, 2020. MMWR Morb Mortal Wkly Rep. 2020;69:477-481. https://doi.org/10.15585/mmwr. mm6915e6.

2. CDC COVID-19 Response Team. Preliminary Estimates of the Prevalence of Selected Underlying Health Conditions Among Patients with Coronavirus Disease 2019 - United States, February 12-March 28, 2020. MMWR Morb Mortal Wkly Rep. 2020;69:382-386. https://doi.org/10.15585/ mmwr.mm6913e2externalicon.

3. Buerhaus PI, Auerbach DI, Staiger DO. Older Clinicians and the Surge in Novel Coronavirus Disease 2019 (COVID-19). JAMA. 2020;323(18):17771778. https://doi.org/10.1001/jama.2020.4978.

4. U.S. Bureau of Labor Statistics. 2010 Standard Occupational Classification System. Available at: https://www.bls.gov/soc/2010/2010_major_ groups.htm\#29-0000. Accessed May 10, 2020.

5. U.S. Bureau of Labor Statistics. Occupational Outlook Handbook: Healthcare Occupations. Available at: https://www.bls.gov/ooh/healthcare/ home.htm. Accessed May 10, 2020.

Publisher's Note: Springer Nature remains neutral with regard to jurisdictional claims in published maps and institutional affiliations. 